\title{
High-resolution structural analysis of a novel octaheme cytochrome $c$ nitrite reductase from the haloalkaliphilic bacterium Thioalkalivibrio nitratireducens
}

Konstantin M. Polyakov ${ }^{\mathrm{a}, \mathrm{b}}$, Konstantin M. Boyko ${ }^{\mathrm{a}}$, Tamara V. Tikhonova ${ }^{\mathrm{a}}$, Alvira Slutsky ${ }^{\mathrm{a}}$, Alexey N. Antipov $^{\mathrm{a}}$, Renata A. Zvyagilskaya ${ }^{\mathrm{a}}$, Alexandre N. Popov ${ }^{\mathrm{c}}$, Gleb Bourenkov ${ }^{\mathrm{c}}$, Viktor S. Lamzin ${ }^{\mathrm{c}}$ and Vladimir O. Popov

\author{
${ }^{a}$ A.N. Bach Institute of Biochemistry, Russian Academy of Sciences, Leninsky pr.33, 119071 \\ Moscow, Russia \\ ${ }^{\mathrm{b}}$ Engelhardt Institute of Molecular Biology, Russian Academy of Sciences, Vavilov str.32, 119991 \\ Moscow, Russia \\ ${ }^{c}$ European Molecular Biology Laboratory (EMBL), c/o DESY, Notkestrasse 85, 22603 Hamburg, \\ Germany \\ Correspondence should be addressed to Prof.Vladimir Popov: vpopov@inbi.ras.ru,
} tel/fax: +7-495-9523441

Running Title: Multi-heme Nitrite Reductase

Keywords: multiheme protein, cytochrome $c$ nitrite reductase, Thiolkalivibrio nitratireducens, 3D structure, electron transport

Bacterial pentaheme cytochrome $c$ nitrite reductases (NrfAs) are key enzymes involved in the terminal step of dissimilatory nitrite reduction of the nitrogen cycle. Their structure and functions are well studied. Recently, a novel octaheme cytochrome $c$ nitrite reductase (TvNiR) has been isolated from the haloalkaliphilic bacterium Thioalkalivibrio nitratireducens. Here we present high-resolution crystal structures of the apo-enzyme and its complexes with the substrate (nitrite) and the inhibitor (azide). Both in the crystalline state and in solution, TvNiR exists as a stable hexamer containing 48 hemes - the largest number of hemes accommodated within one protein molecule known to date. The subunit of TvNiR consists of two domains. The N-terminal domain has a unique fold and contains three hemes. The catalytic C-terminal domain hosts the remaining five hemes, their arrangement, including the catalytic heme, being identical to that found in NrfAs. The complete set of eight hemes forms a spatial pattern characteristic of other multiheme proteins, including structurally characterized octaheme cytochromes. The catalytic machinery of TvNiR resembles that of NrfAs. It comprises the lysine residue at the proximal position of the catalytic heme, the catalytic triad of tyrosine, histidine, and arginine at the distal side, channels for the substrate and product transport with a characteristic gradient of electrostatic potential, and, finally, two conserved $\mathrm{Ca}^{2+}$-binding sites. However, TvNiR has a number of special structural features, including a covalent bond between the catalytic tyrosine and the adjacent cysteine and the unusual topography of the product channels that open into the void interior space of the protein hexamer. The role of these characteristic structural features in the catalysis by this enzyme is discussed. 


\section{Introduction}

Pentaheme cytochrome $c$ nitrite reductases (NrfA proteins) were found in proteobacteria belonging to different subdivisions, ${ }^{1,2}$ including $\gamma$ - (Escherichia coli $^{3}$ ), $\delta$ - (sulfur and sulfate-reducing bacteria Desulfovibrio desulfuricans ${ }^{4,5}$ and Desulfovibrio vulgaris Hildenborough, ${ }^{6-8}$ ) and $\varepsilon^{-}$(Sulfurospirillum deleyianum, Wolinella succinogenes ${ }^{9}{ }^{10}$ ) proteobacteria. They catalyze the six-electron reduction of nitrite to ammonia ${ }^{1,2}$ and play an important role in the biological nitrogen cycle by enabling respiratory nitrite ammonification. ${ }^{1}$ These enzymes also catalyze the production of ammonia through the five-electron reduction of nitric oxide and two-electron reduction of hydroxylamine in the processes that are involved in cellular detoxification. ${ }^{1,2}$ The six-electron reduction of sulfite to sulfide was also reported as the secondary activity of NrfAs. While significantly lower than the specific activities for nitrite reduction, these sulfite reductase activities exceed those found for dissimilatory siroheme-containing sulfite reductases. ${ }^{1,11,12}$

Bacterial NrfAs belong to a well-studied group of enzymes exhibiting similar physiochemical and catalytic properties. ${ }^{1}$ All NrfAs structurally characterized to date ${ }^{3-10}$ are homodimers with each monomer folded as a single domain containing five hemes $c$. The heme arrangement is conserved in all NrfAs. ${ }^{1}$ Four of these hemes have a standard bis-histidine coordination $(\mathrm{CXXCH}$ binding motif in amino acid sequence), whereas the active-site heme is coordinated by lysine (from the CXXCK motif) in the proximal position and water/hydroxyl in the distal one. The active site of NrfAs comprises conserved histidine, tyrosine and arginine residues. Nitrite reductases bind up to two $\mathrm{Ca}^{2+}$ ions, one of which is located close to the enzyme active site and supposed to contribute to its proper structuring. ${ }^{4}$ The molecular mechanism of catalysis of nitrite reduction to ammonia was proposed. ${ }^{13}$ The mechanism involves the alternating electron and proton transfer to the substrate and reaction intermediates bound to the heme iron.

Two other multiheme enzymes - octaheme hydroxylamine oxidoreductase, HAO, ${ }^{14}$ and octaheme tetrathionate reductase, OTR ${ }^{15}$ - were structurally characterized. There is no sequence and structural similarity between these two enzymes and between them and NrfAs. Moreover, OTR exists as a monomer, whereas HAO is a trimer. Nevertheless, these enzymes demonstrate a remarkable similarity in the heme arrangement within the monomers. Seven of eight hemes of OTR and HAO can be superimposed leaving the active site hemes on the opposite sides of the seven-heme chain. All five hemes of NrfA can be almost exactly superimposed on hemes 2-6 of OTR ${ }^{15,16}$ or hemes 4-8 of HAO. ${ }^{10}$ In this case the catalytic hemes form a part of this conserved arrangement. In OTR, like in NrfAs, the catalytic heme is coordinated by lysine in the proximal position. However, as opposed to NrfAs, this residue does not belong to the heme-binding motif (CXXCH in OTR); instead, it is distant along the amino acid sequence. In spite of the complete absence of structural homology between the 
active sites of OTR (probable catalytic residues of OTR are Cys64 and Lys153 ${ }^{15}$ ) and pentaheme nitrite reductases, OTR shows nitrite and hydroxylamine reductase activities, as well as high affinity for nitrite $\left(K_{m} 5 \mu \mathrm{M}\right)$. Hence, the nitrite reduction can be considered as a possible physiological function of OTR in cells. ${ }^{16}$ Unlike NrfAs, OTR does not exhibit sulfite reductase activity but efficiently reduces tetrathionate.

Recently, we have isolated a novel octaheme cytochrome $c$ (TvNiR) with high nitrite reductase activity from the denitrifying sulfur-oxidizing haloalkaliphilic bacterium Thioalkalivibrio nitratireducens. ${ }^{17}$ Like NrfAs, TvNiR catalyzes the reduction of nitrite and hydroxylamine to ammonia without release of any intermediates. In solution TvNiR exists as a stable hexamer containing in total 48 hemes $c{ }^{17,18}$ Despite only $20 \%$ sequence identity with the structurally characterized NrfAs, the catalytically important active-site residues, as well as four CXXCH and one CXXCK heme-binding motifs of the NrfA sequences, are conserved in the TvNiR sequence. TvNiR shows no sequence similarity with HAO or OTR. However, TvNiR displays nearly $50 \%$ identity with eight proteins of the cytochrome $c$ family from $\delta$-proteobacteria (three most homologous of these proteins with SWISS-PROT accession codes: $\underline{\text { Q1NQZ7, }} \underline{\text { A1ATC6 }}$ and Q74G90 are shown on Fig. 1) that comprise eight heme $c$ binding motifs, one of which featuring the characteristic CXXCK motif. ${ }^{19}$ The physiological function of these octaheme cytochromes $c$ remains unclear. It can differ from that of pentaheme nitrite reductases, because at least two of these bacteria (11ATC6 and Q74G90) feature the pentaheme NrfA in their genomes.

As it has been suggested recently ${ }^{20}$ based on the phylogenetic and structural analyses, octaheme cytochrome $c$ nitrite reductases could be possible intermediates in the process of evolution from $\mathrm{N}$ oxide-reducing pentaheme cytochrome $c$ proteins, NrfAs, to N-oxide-oxidizing octaheme proteins related to HAO. The first necessary event in this transition involves the nrfA gene multiplication followed by the N-terminal extension of some of these multiple $n r f A$ genes. However, the structural evolution analysis is of limited value in the absence of three-dimensional structures of octaheme nitrite reductases. A structural insight will allow one to follow the evolution of structure-function relationships for cytochrome $c$ nitrite reductases.

Earlier, we have described the crystallization and preliminary X-ray diffraction study of TvNiR. ${ }^{21} \mathrm{In}$ the present study, we report the high-resolution three-dimensional structures of apo-TvNiR and its two binary complexes with nitrite as the substrate and azide as the inhibitor. The complex quaternary structure and the arrangement of the heme groups were revealed along with novel active-site features. 


\section{Results and discussion}

Subunit structure. The TvNiR monomer comprising 525 amino acid residues is folded into a twodomain structure. The small N-terminal domain (residues 1-69 and 269-287) accommodates the first three hemes (hemes 1 to 3). The much larger catalytic domain (residues 70-268 and 288-525) houses the remaining five hemes, including heme 4 with specific lysine coordination (Fig. 2a, Supplementary Material, Figure S1). The 3D structure of the N-terminal domain consisting of five $\alpha$-helices and two $\beta$-sheets is unique to TvNiR and has no analogs among the known protein folds.

The overall structure of the catalytic domain resembles that of the structurally characterized NrfAs. About $200 \mathrm{C} \alpha$ atoms of the catalytic domain of TvNiR and NrfAs can be superimposed with r.m.s.d. less than $1 \AA$ (Fig. 2b). The superposition includes five hemes from the catalytic domain, as well as 19 out of $30 \mathrm{TvNiR} \alpha$-helices, including three long (22-38 amino acid residues) helices at the C-terminus (Fig. 2b). There is no structural homology between the TvNiR and OTR subunits, whereas the subunit of HAO displays a characteristic bundle of $\alpha$-helices at the C-terminus. The binding of nitrite and azide to the enzyme active site has no effect on the overall subunit structure. The corresponding r.m.s.d. between all $\mathrm{C} \alpha$-atoms of one subunit of the apo- and holo-structures is $c a$. $0.1 \AA$.

Quaternary structure. Both in the crystal structure and in solution, TvNiR monomers form a hexamer (Fig. 3a) with the point group symmetry 32. The overall shape of the TvNiR hexamer can be described as a trigonal bipyramid with characteristic dimensions of $\sim 120 \AA$ (the base) and $\sim 150 \AA$ (the height). The identical monomers of the hexamer are related by a crystallographic threefold axis. The noncrystallographic twofold axis of the hexamer relates the crystallographically independent monomers.

The TvNiR hexamer shows a complex set of intersubunit interactions and can be described as a "dimer of trimers" (Fig. 3a). Each of the TvNiR monomers forms 36 hydrogen bonds with the adjacent monomers within the trimer (the contact surface area per monomer is 3,200 $\AA^{2}$ ) and 9 hydrogen bonds with the adjacent monomers within the dimer (the contact surface area is 1,500 $\AA^{2}$ ). Four additional hydrogen bonds (the contact surface area is $150 \AA^{2}$ ) are formed between the monomers belonging to different dimers and trimers (e.g., the A1-B2 contact). As a result the buried surface area of intersubunit contacts is $4,850 \AA^{2}$ per monomer. All hydrogen bonds that contribute to intersubunit contacts are presented in (Supplementary Material, Table S1).

The interface within the protein trimer is only partially determined by the long C-terminal $\alpha$-helices, which can be considered as the major secondary structural motif of the subunit. In NrfAs and HAO, the role of these $\mathrm{C}$-terminal $\alpha$-helices in the formation of the quaternary structure is much more profound. Extensive contacts between these helices result in dimerization of the subunits in NrfAs and trimerization in HAO. The buried surface area per subunit in the NrfA dimers is considerably smaller 
than that in TvNiR and allows them to dissociate under conventional conditions. The enzyme activity of NrfA from S. deleyianum (the buried surface area is $2,150 \AA^{2}$, which is 2.2 -fold smaller than that for TvNiR) was shown to depend on the protein concentration with the characteristic constant $\mathrm{K}_{\mathrm{d}}=1$ $3 \mathrm{nM},{ }^{22}$ whereas the dissociation constant of the enzyme from E. coli with a smaller contact surface $\left(1,530 \AA^{2}\right)$ is much higher $(4 \mu \mathrm{M}) .{ }^{12}$ Very tight intersubunit contacts are observed in the HAO trimer $\left(5,427 \AA^{2}\right)$, which is additionally stabilized by a covalent link between the catalytic heme and the Tyr467 residue from the adjacent subunit. ${ }^{14}$ As a result of formation of the hexameric structure, the buried surface area of TvNiR $\left(4,850 \AA^{2}\right)$ is close to that of HAO. This fact accounts for extremely high resistance of the enzyme to dissociation. ${ }^{18}$ The dissociation of the TvNiR hexamer in solution was observed only in the presence of urea and guanidine chloride at high concentrations. In accordance with the relative contributions of the dimeric and trimeric contacts to the formation of the native structure, ${ }^{17,18}$ the dissociation proceeds at the dimeric contact to form trimers followed by dissociation of the latter into monomers, no formation of dimers being observed during dissociation of TvNiR.

A specific feature of the TvNiR hexamer is the presence of a large cavity in the center of the molecule (Fig. 3a, b). The shape of the inner cavity can best be described as three intersecting spheres. One large sphere with a radius of $19 \AA$ is located in the center of the hexamer, and two small spheres with a radius of $12 \AA$ each are located inside the trimers (Fig. 3b). The distances between the centers of the large and small spheres are $17 \AA$. The walls of the cavity within the trimer are formed by the residues $82-95,114-116,402-404(\alpha 26)$, and 421-435 ( $\alpha 28)$ of each subunit comprising the trimer. The walls of the large cavity are formed by the residues 83 , 404-408, 419-421, and 486-488 and the propionate groups of hemes 6 and 7 from all the subunits. The intersection of two spheres makes the "nozzle," that is formed by the negatively charged amino acid residues Glu83, Asp405, Glu420, and Asp 421 of each monomer (Fig. 3c). This nozzle with a diameter about $17 \AA$ enables exchange of molecules between the cavity in the trimer and the large cavity. The latter is, in turn, accessible to the bulk solvent through three oval inlets/outlets of size 9 × $16 \AA$ located along the non-crystallographic axes of the hexamer (Fig. 3a, d).

Active site. The TvNiR active site is buried $10 \AA$ from the protein surface and incorporates heme 4 . The iron atom in the catalytic heme is axially coordinated by Lys188 from the CXXCK heme-binding motif (Fig. 4a-c). A similar coordination is observed for the catalytic hemes in NrfAs. However, the distance between the NZ atom of Lys188 and the catalytic Fe ion is $c a .1 .9 \AA$ in apo-TvNiR and remains unaltered in the complexes with both the substrate (nitrite) and the inhibitor (azide) (Supplementary Material, Table S2). This distance is slightly shorter than that found in NrfAs (2.1 $\AA),{ }^{10,13}$ which may be of significance for the efficient catalytic turnover of the enzyme. In the apo- 
structure, the heme 4 distal site is occupied by a water molecule (Fig. $4 \mathrm{a}$ ). The distance between the oxygen atom of the water molecule and $\mathrm{Fe}$ is $2.1 \AA$. In the complexes (Fig. 4b, c), nitrite is coordinated to the catalytic heme via the nitrogen atom; azide, via the terminal N1 atom. In both complexes, the distance to the catalytic Fe atom is $\sim 1.9 \AA$. It should be noted that the inhibitor (azide ion) binding in TvNiR differs from that in $\mathrm{NrfA},{ }^{9}$ in which azide does not interact with the iron of the catalytic heme but is bound in the substrate channel close to the entrance to the active site.

The residues Arg131, Tyr303, and His361 are in the immediate vicinity of the catalytic Fe ion. The residues Tyr303 and His361 are involved in hydrogen bonding with the bound water molecule in the apo-structure and with nitrite and azide in the structures of the complexes. In addition, the substrate and the inhibitor form a hydrogen bond with Arg131 (Fig. 4a-c and Supplementary Material, Table S2). The hydrogen bonds formed by the tyrosine and histidine residues with the bound anions are shorter than the hydrogen bonds formed by the arginine residue. It is in accord with the assumed role of the conserved active site residues in NrfAs, ${ }^{9,10,13}$ where Tyr303 and His361 form a part of the catalytic machinery, but Arg131 plays mainly the coordinating role by properly positioning the substrates and the putative catalytic intermediates at the catalytic Fe ion. In addition, the active site cavity is formed by Phe109, Lys358, Gln360, and the main chain of Leu359. Due to the presence of charged residues, the enzyme active site has a strong positive potential, thus facilitating the binding of the negatively charged substrate and the release of the positively charged reaction product (ammonium ion). All active site residues are conserved in NrfAs, as well as in other multi-heme cytochromes homologous to TvNiR (Fig. 1).

TvNiR displays essentially the same architecture of the enzyme active site as the NrfAs. The spatial alignment of the catalytic hemes gives r.m.s.d. of approximately $0.2 \AA$ and results in almost the precise superposition of the active site residues Arg131, His361, and Lys188 (r.m.s.d. is $0.2 \AA$ ) and a significant difference in position of Tyr303. In TvNiR the side chain of Tyr303 is shifted (the distance between the corresponding $\mathrm{OH}$ atoms is $1.2 \AA$ ) closer to the water molecule coordinated by the active site Fe atom with respect to the site found in NrfAs. This shift is apparently attributed to a covalent bond between the ortho position of Tyr303 and the S atom of Cys305, which is clearly seen in electron density maps (Fig. 4d). The Cys residue was also revealed in the amino acid sequences of multi-heme cytochromes that share the highest homology with $\mathrm{TvNiR}^{17}$ (Fig. 1). NrfAs lack such a covalent bond since Cys305 is substituted by phenylalanine in their sequences.

The formation of this internal link has two major consequences for the TvNiR active site structure and properties. First, it causes a change in the orientation of the tyrosine ring and leads to the overall compaction of the TvNiR active site. As a result, in the complex of TvNiR with nitrite, the hydrogen bonds formed by His361 and Tyr303 with the oxygen atom of the bound substrate molecule are 
substantially shorter (2.4 and $2.8 \AA$ respectively) than those in the enzyme from $W$. succinogenes $(2.6$ and $3.8 \AA$, respectively). ${ }^{13}$ Second, the formation of the cysteine-tyrosine covalent bond results in a lowering of $\mathrm{pK}_{\mathrm{a}}$ of the hydroxyl group of Tyr303 by $0.5-1.0$ units, ${ }^{23-25}$ thus making it a much more efficient proton donor. Apparently, these changes in the orientation and properties of Tyr303 are responsible for the higher catalytic activity of $\mathrm{TvNiR}$ in the nitrite reduction $\left(13 \cdot 10^{3} \mathrm{e}^{-} \mathrm{s}^{-1}\right.$ per active centre) compared to NrfAs $\left(4-8 \cdot 10^{3} \mathrm{e}^{-} \mathrm{s}^{-1}\right) .{ }^{17}$ The importance of the active site tyrosine residue for the catalysis by W. succinogenes NrfA has been confirmed in the recent study, ${ }^{11}$ where the replacement of this residue by phenylalanine was shown to lead to the virtually complete loss of nitrite reductase activity, structural changes in the active site being minor. It is worth noting that Tyr218 in this enzyme is not directly involved in hydrogen bonding with the nitrite molecule (the distance is $3.8 \AA$ ).

TvNiR provides a rare example of a tyrosine-cysteine covalent bond in the active sites of the enzymes. Similar covalent bonds have been earlier reported only for siroheme-containing sulfite reductase ${ }^{26}$ and galactose oxidase ${ }^{23,27}$ and are considered to be a specific fingerprint of sulfite reduction in sirohemecontaining reductases. If the bond is artificially deleted by site-directed mutagenesis, the substrate preference shifts towards nitrite reduction. ${ }^{26}$ The specific features of the internal architecture of the TvNiR active site suggest that the enzyme in vivo could have another function besides nitrite reduction, e.g. sulfite reduction.

A number of well-structured water molecules that are present in the active site form a complex hydrogen bond network between each other and the active site residues (Supplementary Material, Table S2).

The active site of TvNiR, like that in NrfAs, is connected to the bulk via two channels serving for the substrate entry and product release. The residues delineating the channels are listed in (Supplementary Material, Table S3). The substrate channel has a diameter of $\sim 6 \AA$. The chain consisting of five water molecules located in the channel leads to the protein surface. The distances between these waters do not exceed $3.2 \AA$, and these water molecules are hydrogen-bonded to protein side chains and other water molecules (Supplementary Material, Table S3). The water molecule immediately adjacent to the enzyme active site is hydrogen-bonded to the amide group of Gln360 and the hydroxyl group of the catalytic Tyr303. The entrance to the channel has a positive surface charge due to the presence of Arg171, Arg316, and Arg348 (Fig. 5) that can facilitate transport of the negatively charged substrate to the enzyme active site. The center of the channel is characterized by a nearly neutral potential with a negative patch around Asp346. This residue, conserved in NrfAs, is located in a niche of the channel wall and can push the negatively charged substrate directly to the substrate site, where the residues Arg131 and His361 can attract and properly position the substrate molecule. 
The product egress channel has a slightly smaller diameter ( $5 \AA$ ) and is occupied by six hydrogenbonded water molecules with water-to-water distances shorter than $3.5 \AA$. The product channel is strongly negative at the entry to the enzyme active site due to the presence of Glu115 and the propionate moieties of heme 4 (Fig. 5, Supplementary Material, Figure S2). This is important for transport of the positively charged reaction product (ammonium ion). The negative charge within the channel is provided by the propionate groups of hemes 6 and 7 and the residues Glu83, Glu115, and Asn486 (Supplementary Material, Table S4). Surprisingly, there is a strong positively charged area formed by Arg81, His85, Arg96, Arg404, and Lys431 at the exit of the channel. The last three residues are conserved in octaheme cytochromes homologous to TvNiR (Fig. 1), which confirms the importance of this positively charged area for enzyme functioning. Due to the specific quaternary structure of TvNiR, the outlet of the product channel in this enzyme leads not to the protein surface, as observed in NrfAs, but to the inner cavity of the trimer (Fig. 3b). As described above, this cavity is connected to the internal space in the center of the hexamer and further with the bulk through the negatively charged outlet formed by Glu83, Asp405, Glu 420, and Asp421 that can facilitate ejection of the product from the interior of the protein. To the best of our knowledge, TvNiR is the only example of enzymes with such a peculiar arrangement for the product 'disposal'.

Nevertheless, the overall charge distribution along the substrate and product channels in TvNiR follows the same rule as in NrfAs. The positively charged substrate channel and the active site facilitate substrate delivery; the negatively charged product channel, the product release from the enzyme active site.

In TvNiR the efficient proton transport mechanism should function to service the catalytic machinery of the enzyme. To perform a single catalytic turnover eight protons should be delivered to the nitrite reductase active site. This is presumably achieved through a network of water molecules occupying the substrate and/or product channels. The extension of the network of interacting water molecules (5 molecules for the substrate channel and 6 molecules for the product channel) favors proton transport through the substrate channel. On the other hand, the product channel has a much more favorable charge distribution. However, it could be the case that both the substrate and product channels are employed to deliver protons to the catalytic site. 
Ca ions. The apo-TvNiR reveals one firmly bound $\mathrm{Ca}^{2+}$ ion per monomer. It is located close to the active site with the Ca-Fe distance of $10.5 \AA$. The $\mathrm{Ca}^{2+}$-binding site is in an octahedral coordination environment formed by the residues Glu302, Tyr303, Lys358, and Gln360 conserved in NrfAs and two water molecules (Supplementary Material, Table S4). By analogy with NrfAs, ${ }^{4-11}$ this $\mathrm{Ca}^{2+}$ ion is suggested to play an important role in the structuring of the enzyme active site (Fig. 6a).

The secondary $\mathrm{Ca}^{2+}$-binding site was found in NrfAs from D. desulfuricans, W. succinogenes, E. coli and D. vulgaris Hildenborough. ${ }^{4,6,9,10}$ It is situated in the vicinity of hemes 6 and 7 (TvNiR numbering), usually has an incomplete coordination sphere, except for $D$. desulfuricans, and is bound to the propionate side chains of these hemes. In contrast to the first $\mathrm{Ca}^{2+}$-binding site, the second site is labile. There is no metal ions in this position in the enzyme from $D$. deleyianum, ${ }^{10}$ and the $\mathrm{Ca}^{2+}$ ion can be exchanged for other cations present in solution, e.g., yttrium in W. succinogenes NrfA. ${ }^{9}$

The second bound $\mathrm{Ca}^{2+}$ ion is absent in the apo-TvNiR structure; however, this ion was found (with 0.5 occupancy) in the structures of both complexes (Fig. 6b). Like in other NrfAs, this $\mathrm{Ca}^{2+}$-binding site has an incomplete coordination sphere and is bound to the propionate groups of hemes 6 and 7 and the main chain of Pro116. In the structure of the complexes, one of the propionate groups of heme 7 has two conformations (with 0.5 occupancy each). One of them is similar to the conformation in the free enzyme (without bound $\mathrm{Ca}^{2+}$ ), whereas the second conformation is similar to that in the structure of NrfAs with the bound second $\mathrm{Ca}^{2+}$ ion. The $\mathrm{Ca}^{2+}$ coordination sphere is completed by binding three water molecules. When the NrfA hemes are superimposed on those of TvNiR, both the primary and secondary $\mathrm{Ca}^{2+}$-binding sites also superimpose. In $\mathrm{TvNiR}$ the second $\mathrm{Ca}^{2+}$ ion is located within the putative product egress channel, thus blocking the release of the reaction product into the bulk. A similar situation is observed in NrfA from D. desulfuricans. ${ }^{4}$ As opposed to NrfAs, ${ }^{22}$ the TvNir activity is affected neither by endogenous calcium, nor by chelating agents.

Heme arrangement. The hexamer of TvNiR contains altogether 48 hemes $c$. Eight hemes in each TvNiR monomer are located within 9-11 A from each other, presumably enabling efficient electron exchange. Within each monomer the hemes are arranged pairwise according to the classical "di-heme elbow" and "parallel stacking" motifs. ${ }^{28}$ The planes of hemes 2-3, 5-6, and 7-8 are nearly perpendicular to each other, whereas the planes of hemes 1-2, 3-5, and 6-7 are nearly parallel. The only heme that is not involved in these patterns is catalytic heme 4 (Fig. 7a).

Eight hemes of TvNiR display the arrangement shared with other proteins containing multiple hemes c. Hemes 4 to 8 can be superimposed on the five hemes of NrfAs with r.m.s.d. ranging from $0.5 \AA$ for the enzyme from $D$. deleyianum to $0.6 \AA$ for the enzyme from $D$. desulphuricans, whereas all eight hemes of TvNiR match eight hemes of HAO with r.m.s.d. of $1.3 \AA$ (Fig. 7a). The significance of the alignment is additionally confirmed by superposition of the catalytic hemes of these enzymes. 
For OTR, the alignment can be made in two ways. The one favored by the authors of the original paper aligns seven hemes of HAO with seven hemes of OTR leaving the catalytic hemes off the alignment on the opposite sides of the heme chain. ${ }^{15}$ This alignment results in r.m.s.d. of $3.4 \AA$ for both HAO and TvNiR. However, there is another option, which involves the alignment of only six core hemes (Fig. 7a). The results of this superimposition are presented in Fig. 7a. This mode of alignment keeps the catalytic hemes of all the enzymes in the same place and results in r.m.s.d of 2.3$2.4 \AA$ for the OTR-HAO and OTR-TvNiR pairs (Supplementary Material, Table S5).

The hexameric structure adopted by TvNiR does not result in the formation of an extended network of interacting hemes (Fig. 7b, c). The closest interheme distance within the hexamer enabling heme interactions is observed at the dimer interface between hemes 3 of the adjacent subunits belonging to different trimers (Fig. 7b). The Fe to Fe distance between these hemes is $13.1 \AA$, whereas the edge to edge distance is $4.1 \AA$. In the TvNiR trimers, the edge to edge distance between the nearest hemes 6 and 8 belonging to different monomers is about $18 \AA$ and prohibits direct interactions between the hemes (Fig. 7c). Thereby, in the TvNiR hexamer, 48 hemes are clustered into three isolated groups, each comprising 16 interacting hemes. There is an apparent contradiction between the highly stable quaternary structure, very efficient catalysis ( $\mathrm{TvNiR}$ is the fastest nitrite reductase known to date), and a lack of benefit from forming such a complex structure in terms of heme-heme cooperativity.

This is in contrast to extended heme arrays generated by the symmetrical quaternary structures of NrfAs and HAO, each resulting in unique ensembles of interacting hemes (10 for NrfAs ${ }^{10}$ and 24 for $\mathrm{HAO}^{14}$ ). Moreover, the formation of stable or transient multiheme complexes containing electron donors, such as $\mathrm{NrfA}_{2} \mathrm{~B}_{2}$ from E.coli ${ }^{29}$ or $\mathrm{NrfA}_{4} \mathrm{H}_{2}$ from D. vulgaris, ${ }^{6}$ results in extension of the network of interacting hemes even further to 20 and 28 hemes, respectively.

Modeling of a putative physiological donor-binding site. The nature of the physiological electron donor of TvNiR is unknown. However, it is known that $\mathrm{NrfH}$ (membrane-bound tetraheme-containing cytochrome) is the physiological electron donor for NrfA from $\varepsilon$ - and $\delta$-proteobacteria, and NrfB (water-soluble pentaheme c-type cytochrome) is the physiological electron donor for NrfA from $\gamma$ proteobacteria. The three-dimensional structure of the NrfH-NrfA complex was established. ${ }^{6}$ In this complex, the mutual arrangement of the dimeric NrfH molecule and two dimeric NrfA molecules enables the direct electron transfer between hemes 4 of the NrfH molecule and one of hemes 5 of the NrfA dimer. The three-dimensional structure of NrfB was also determined. ${ }^{29}$ The authors of this study modeled the NrfB-NrfA complex, in which the direct electron transfer can occur between heme 1 of NrfA and heme 5 of NrfB, hemes 1 and 2 of NrfB being presumably responsible for the binding of the NrfB-NrfA complex with the reducing membrane-bound NrfC-NrfD complex. 
To locate the putative donor-binding site in the TvNiR hexamer, the accessibility of individual hemes was estimated. Four hemes in the TvNiR hexamer (hemes 1, 3, 5, and 8) have an accessible surface area in excess of $95 \AA^{2}$ when probed with a water molecule. However, with the probe radius increased to 5 and further to $15 \AA$ (the latter case is a more realistic situation for a small protein - a putative physiological electron donor of TVNiR) only one heme (heme 8) still has the accessible surface area of $77 \AA^{2}$ (Supplementary Material, Table S6). Therefore, the presumable donor-binding site in the TvNiR hexamer is located in the channel, which runs along the twofold axis of the hexamer and connects the inner cavity of the hexamer to the bulk solvent (Fig. 3d). Two hemes 8 from different protein subunits related by the non-crystallographic twofold axis (e.g., A1 and B2 in Fig. 3d) are exposed to this area with the distance between them of about $30 \AA$.

An attempt was made to manually model the binding of the NrfH molecule to the TvNiR hexamer in the vicinity of heme 8 . For steric reasons, the $\mathrm{NrfH}$ dimer (or monomer) cannot be docked to the TvNiR hexamer in such a way that the arrangement of heme 4 of $\mathrm{NrfH}$ with respect to heme 8 of TvNiR would be analogous to their arrangement in the structure of the NrfH-NrfA complex, ${ }^{6}$ in which these hemes are perpendicular to each other. Nevertheless, the $\mathrm{NrfH}$ dimer can be located (without substantial steric hindrance) on the non-crystallographic twofold axis at the entrance to the channel that connects the inner cavity of the hexamer to the bulk solvent in a such a way that one of hemes 4 of $\mathrm{NrfH}$ is parallel to and is at an interacting distance from heme 8 of the subunit A1 and another heme 4 is parallel to and is at an interacting distance from heme 8 of the subunit B2 (Fig. 8). Assuming that a molecule similar to $\mathrm{NrfH}$ from D. vulgaris serves as the physiological donor for TvNiR and that the proposed model corresponds to the structure of the TvNiR complex with its physiological donor, this binding mode of the electron donor results in the formation of an electrontransport chain comprising hemes from four of the six TvNiR monomers.

It should be noted that the NrfB molecule in the structural model of the NrfB - NrfA complex ${ }^{29}$ occupies the same relative spatial position as the $\mathrm{N}$-terminal domain in the TvNiR structure. Hemes 1 and 2 of NrfB in the model complex are exposed to the bulk solvent and are thought to enable binding to another electron carrier, NrfC. ${ }^{1,29}$ When hemes 1 and 2 of NrfB are superimposed on hemes 1 and 2 of TvNiR (r.m.s.d $0.8 \AA$ ), not only the heme atoms but also the adjacent protein chains are properly superimposed (r.m.s.d. $0.8 \AA$ ). Thus, the N-terminal domain of TvNiR with its three hemes extra to the five hemes found in NrfAs can be considered as a part of the putative electron transmitting protein, analogue of NrfB, which is, however, covalently fused into the TvNiR.

\section{Conclusion}


The octaheme cytochrome from $T v$. nitratireducens displays many structural and functional features specific to pentaheme nitrite reductases, NrfAs, from proteobacteria. It catalyzes $6 e$ reduction of nitrite to ammonia. About 50\% (46-54) of the amino acid residues of its catalytic domain occupy positions structurally equivalent to the positions of the residues in NrfAs. The TvNiR reveals a canonical arrangement of the enzyme active site comprising heme $c$ ligated by lysine at the proximal side and displaying a catalytic triad composed of tyrosine, histidine, and arginine at the distal side. The enzyme is equipped with channels for substrate transport and product release with a characteristic gradient of electrostatic potential, shows two conserved $\mathrm{Ca}^{2+}$-binding sites, and reveals a conserved arrangement of five hemes $c$ within the monomer with the catalytic heme occupying the same position as in NrfAs.

However, several important features differentiate TvNiR from the NrfAs. First, there is a covalent bond between the catalytic tyrosine and the adjacent cysteine in the enzyme active site. Second, TvNiR contains additional three hemes $c$ organized in a special $\mathrm{N}$-terminal domain with a unique fold. Third, this enzyme forms a highly stable hexameric structure both in the crystalline state and in solution, resulting in a rather specific channeling of the product inside the interior of the protein. And finally, the structure of TVNiR is the first example of the enzyme comprising 48 hemes $c$. This is the largest absolute number of hemes accommodated within one protein molecule known to date.

A number of further questions arise from the deduced three-dimensional structure of TvNiR: Why so many hemes are required? What are the benefits of formation of this complex and highly symmetrical structure besides high protein stability and how are they realized? What is the putative physiological electron donor for nitrite reduction? What is the structure of the complex with the donor? Many other questions also await further research.

\section{Methods}

Amino acid alignment. The pairwise and multiple amino acid alignments were performed as described earlier. $^{21}$

Crystallization, data collection, structure determination and refinement. The protein was purified as was described in. ${ }^{10}$ Crystallization, data collection, and TvNiR structure determination and refinement were carried out as was described in. ${ }^{21}$ The structure of the oxidized form of apo-TvNiR was solved by the SAD technique. ${ }^{21}$

The data collection and refinement statistics are listed in Table 1. There are two crystallographically independent polypeptide chains per asymmetric unit related by a non-crystallographic twofold axis. 
The first five residues and the last residue in the polypeptide chain of apo-TvNiR are not visible in electron density maps; 58 residues have multiple conformations.

The final $1.5 \AA$ resolution TvNiR model (520 residues and 8 hemes) was refined with the REFMAC $\operatorname{program}^{30}$ to $\mathrm{R}_{\mathrm{f}}=0.126$ and $\mathrm{R}_{\text {free }}=0.141($ Table 1$)$.

Crystals of the TvNiR complexes with the substrate (nitrite) and the inhibitor (azide) were obtained by soaking of the apo-enzyme crystals (one hour for the nitrite complex and 12 hours for the azide complex), which were grown as was described in, ${ }^{21}$ in a solution of the ligands in the reservoir buffer. The ligand concentration was $100 \mathrm{mM}$ for nitrite and $10 \mathrm{mM}$ for azide. The X-ray data sets were collected at $100 \mathrm{~K}$ on the X13 beamline at the EMBL Hamburg Outstation. The data were processed as described for apo-TvNiR. All crystals belong to the space group P2 ${ }_{1} 3$ and have similar unit cell parameters. The structures of both complexes were refined with the REFMAC program to $R_{f}=0.149$ $\left(\mathrm{R}_{\text {free }}=0.166\right)$ at $1.8 \AA$ for nitrite and to $\mathrm{R}_{\mathrm{f}}=0.158\left(\mathrm{R}_{\text {free }}=0.174\right)$ at $1.7 \AA$ for azide. The manual refinement was done with the $O^{31}$ and COOT programs. ${ }^{32}$ The data collection and refinement statistics for the complexes are listed in Table 1. The complexes contain 518 and 519 amino acid residues in the A and B subunits, respectively, and eight hemes per subunit. In both complexes, four N-terminal and two C-terminal residues of the subunit $\mathrm{A}$, as well as four $\mathrm{N}$-terminal residues and one C-terminal residue of the B subunit, are not visible in electron density maps.

The occupancies of both the substrate (nitrite) and the inhibitor (azide) molecules in the structures of the complexes are 0.5 . In both complexes, the active site contains a water molecule with an occupancy of 0.5 , whose position is similar to that of the water molecule in the apo-enzyme. Therefore, the structures of the complexes were modeled as a mixture of the complex containing the bound substrate or inhibitor and the complex with a water molecule (apo-enzyme) at half occupancy.

Structure analysis. The 3D structure analysis of the $\mathrm{N}$-terminal and catalytic domains of TvNiR was carried out with the TOPP program ${ }^{33}$ and using the SCOPE database. ${ }^{34}$ The monomers from the TvNiR asymmetric unit as well as the TvNiR, NrfAs, HAO, and OTR monomers were superimposed with the use of the LSQKAB program. ${ }^{35}$ The superposition of the active sites was performed using the same program. The calcium ion in the TvNiR structure was identified by the bond-valence method ${ }^{36}$. The contacts between the subunits in the TVNiR hexamer were analyzed with the CONTACT and AREAIMOL programs ${ }^{37}$ from the CCP4 suite $^{38}$. The former program was used to explore hydrogen bonds between the subunits; the latter program, for the estimation of the contact area surface as well as of the accessible surface area of the hemes in the hexamer. 
The CCP4mg ${ }^{39}$ v.0.12 and PyMol v.0.99rc2 [http://www.pymol.org] programs were used for visualization, electrostatic potential calculations, and investigation of the inner cavity in the hexamer and of the substrate and product channels.

Accession codes. Protein Data Bank: atomic coordinates have been deposited with the accession codes $2 \mathrm{OT} 4$ for the apo-TvNiR, 3D1I for the complex with nitrite, and 2ZO5 for the complex with azide.

\section{Acknowledgements}

We thank Alexey Filimonenkov from the Bach Institute of Biochemistry of the Russian Academy of Sciences (Moscow) for assistance in protein purification. Special thanks are for Dr. Julea Butt from the University of East Anglia (UK) and Dr. Tatiana Safonova from the Bach Institute of Biochemistry of the Russian Academy of Sciences (Moscow) for fruitful discussions and help in manuscript preparation.

The work was supported by the grants of RFBR (No 08-04-00830-a and 07-04-01559-a) and the Federal Agency for Science and Innovations (No 02.512.12.2002). 


\section{References}

1. Simon, J. (2002). Enzymology and bioenergetics of respiratory nitrite ammonification. FEMS Microbiol. Rev. 26, 285-309.

2. Richardson, D. J. \& Watmough, N. J. (1999). Inorganic nitrogen metabolism in bacteria. Curr. Opin. Chem .Biol. 3, 207-219.

3. Bamford, V. A., Angove, H. C., Seward, H. E., Thomson, A. J., Cole, J. A., Butt, J. N. et al. (2002). Structure and spectroscopy of the periplasmic cytochrome c nitrite reductase from Escherichia coli. Biochemistry, 41, 2921-2931.

4. Cunha, C. A., Macieira, S., Dias, J. M., Almeida, G., Goncalves, L. L., Costa, C., Lampreia, J. et al. (2003). Cytochrome c nitrite reductase from Desulfovibrio desulfuricans ATCC 27774. The relevance of the two calcium sites in the structure of the catalytic subunit (NrfA). J. Biol. Chem. 278, 17455-17465.

5. Almeida, M. G., Macieira, S., Goncalves, L. L., Huber, R., Cunha, C. A., Romao, M. J. et al. (2003). The isolation and characterization of cytochrome c nitrite reductase subunits (NrfA and NrfH) from Desulfovibrio desulfuricans ATCC 27774. Re-evaluation of the spectroscopic data and redox properties. Eur. J. Biochem. 270, 3904-3915.

6. Rodrigues, M. L., Oliveira, T. F., Pereira, I. A. \& Archer, M. (2006). X-ray structure of the membrane-bound cytochrome c quinol dehydrogenase $\mathrm{NrfH}$ reveals novel haem coordination. EMBO J. 25, 5951-5960.

7. Rodrigues, M. L., Oliveira, T., Matias, P. M., Martins, I. C., Valente, F. M., Pereira, I. A. \& Archer, M. (2006). Crystallization and preliminary structure determination of the membrane-bound complex cytochrome c nitrite reductase from Desulfovibrio vulgaris Hildenborough. Acta Crystallogr., Sect. F: Struct. Biol. Cryst. Commun. 62, 565-568.

8. Pereira, I. A., LeGall, J., Xavier, A. V. \& Teixeira, M. (2000). Characterization of a heme c nitrite reductase from a non-ammonifying microorganism, Desulfovibrio vulgaris Hildenborough. Biochim. Biophys. Acta, 1481, 119-130.

9. Einsle, O., Stach, P., Messerschmidt, A., Simon, J., Kroger, A., Huber, R. \& Kroneck, P. M. (2000). Cytochrome c nitrite reductase from Wolinella succinogenes. Structure at 1.6 A resolution, inhibitor binding, and heme-packing motifs. J. Biol. Chem. 275, 3960839616.

10. Einsle, O., Messerschmidt, A., Stach, P., Bourenkov, G. P., Bartunik, H. D., Huber, R. \& Kroneck, P. M. (1999). Structure of cytochrome c nitrite reductase. Nature, 400, 476480.

11. Lukat, P., Rudolf, M., Stach, P., Messerschmidt, A., Kroneck, P. M., Simon, J. \& Einsle, O. (2008). Binding and reduction of sulfite by cytochrome $\mathrm{c}$ nitrite reductase. Biochemistry, 47, 2080-2086.

12. Clarke, T. A., Hemmings, A. M., Burlat, B., Butt, J. N., Cole, J. A. \& Richardson, D. J. (2006). Comparison of the structural and kinetic properties of the cytochrome c nitrite reductases from Escherichia coli, Wolinella succinogenes, Sulfurospirillum deleyianum and Desulfovibrio desulfuricans. Biochem. Soc. Trans. 34, 143-145.

13. Einsle, O., Messerschmidt, A., Huber, R., Kroneck, P. M. \& Neese, F. (2002). Mechanism of the six-electron reduction of nitrite to ammonia by cytochrome c nitrite reductase. J. Am. Chem. Soc. 124, 11737-11745.

14. Igarashi, N., Moriyama, H., Fujiwara, T., Fukumori, Y. \& Tanaka, N. (1997). The 2.8 A structure of hydroxylamine oxidoreductase from a nitrifying chemoautotrophic bacterium, Nitrosomonas europaea. Nat. Struct. Biol. 4, 276-284. 
15. Mowat, C. G., Rothery, E., Miles, C. S., McIver, L., Doherty, M. K., Drewette, K. et al. (2004). Octaheme tetrathionate reductase is a respiratory enzyme with novel heme ligation. Nat. Struct. Mol. Biol. 11, 1023-1024.

16. Atkinson, S. J., Mowat, C. G., Reid, G. A. \& Chapman, S. K. (2007). An octaheme ctype cytochrome from Shewanella oneidensis can reduce nitrite and hydroxylamine. FEBS Lett. 581, 3805-3808.

17. Tikhonova, T. V., Slutsky, A., Antipov, A. N., Boyko, K. M., Polyakov, K. M., Sorokin, D. Y. et al. (2006). Molecular and catalytic properties of a novel cytochrome c nitrite reductase from nitrate-reducing haloalkaliphilic sulfur-oxidizing bacterium Thioalkalivibrio nitratireducens. Biochim. Biophys. Acta 1764, 715-723.

18. Tikhonova, T. V., Slutskaya, E. S., Filimonenkov, A. A., Boyko, K. M., Kleimenov, S. Y., Konarev, P. V. et al. (2008). Isolation and oligomeric composition of cytochrome c nitrite reductase from the haloalkaliphilic bacterium Thioalkalivibrio nitratireducens. Biochemistry (Mosc.), 73, 164-170.

19. Methe, B. A., Nelson, K. E., Eisen, J. A., Paulsen, I. T., Nelson, W., Heidelberg, J. F. et al. (2003). Genome of Geobacter sulfurreducens: metal reduction in subsurface environments. Science, 302, 1967-1969.

20. Klotz, M. G., Schmid, M. C., Strous, M., op den Camp, H. J., Jetten, M. S. \& Hooper, A. B. (2008). Evolution of an octahaem cytochrome c protein family that is key to aerobic and anaerobic ammonia oxidation by bacteria. Environ. Microbiol. 10, 3150-3163.

21. Boyko, K. M., Polyakov, K. M., Tikhonova, T. V., Slutsky, A., Antipov, A. N., Zvyagilskaya, R. A. et al. (2006). Crystallization and preliminary X-ray analysis of cytochrome c nitrite reductase from Thioalkalivibrio nitratireducens. Acta Crystallogr., Sect.F: Struct. Biol. Cryst. Commun. 62, 215-217.

22. Stach, P., Einsle, O., Schumacher, W., Kurun, E. \& Kroneck, P. M. (2000). Bacterial cytochrome c nitrite reductase: new structural and functional aspects. J. Inorg. Biochem. 79, 381-385.

23. Whittaker, M. M., Chuang, Y. Y. \& Whittaker, J. W. (1993). Models for the redox active site in galactose oxidase. J. Am. Chem. Soc. 115, 10029-10035.

24. Himo, F., Noodleman, L., Blomberg, M. R. A. \& Siegbahn, P. E. M. (2002). Relative acidities of ortho-substituted phenols, as model for modified tyrosines in proteins. $J$. Phys. Chem. 106, 8757-8761.

25. Itoh, S., Takayama, S., Arakawa, R., Furuta, A., Komatsu, M., Ishida, A., Takamuku, S. \& Fukuzumi, S. (1997). Active site models for galactose oxidase. Electronic effect of the thioether group in the novel organic cofactor. Inorg. Chem. 36, 1407-1416.

26. Schnell, R., Sandalova, T., Hellman, U., Lindqvist, Y. \& Schneider, G. (2005). Siroheme- and [Fe4-S4]-dependent NirA from Mycobacterium tuberculosis is a sulfite reductase with a covalent Cys-Tyr bond in the active site. J. Biol. Chem. 280, 2731927328.

27. Ito, N., Phillips, S. E., Stevens, C., Ogel, Z. B., McPherson, M. J., Keen, J. N. et al. (1991). Novel thioether bond revealed by a 1.7 A crystal structure of galactose oxidase. Nature, 350, 87-90.

28. Mowat, C. G. \& Chapman, S. K. (2005). Multi-heme cytochromes--new structures, new chemistry. Dalton Trans, 3381-3389.

29. Clarke, T. A., Cole, J. A., Richardson, D. J. \& Hemmings, A. M. (2007). The crystal structure of the pentahaem c-type cytochrome NrfB and characterization of its solutionstate interaction with the pentahaem nitrite reductase NrfA. Biochem. J. 406, 19-30. 
30. Murshudov, G. N., Vagin, A. A. \& Dodson, E. J. (1997). Refinement of macromolecular structures by the maximum-likelihood method. Acta Crystallogr., Sect. D: Biol. Crystallogr. 53, 240-255.

31. Jones, T. A., Zou, J. Y., Cowan, S. W. \& Kjeldgaard, M. (1991). Improved methods for building protein models in electron density maps and the location of errors in these models. Acta Crystallogr., Sect. A, 47 ( Pt 2), 110-9.

32. Emsley, P. \& Cowtan, K. (2004). Coot: model-building tools for molecular graphics. Acta Crystallogr., Sect. D: Biol Crystallogr 60, 2126-2132.

33. Lu, G. (1996). A WWW service system for automatic comparison of protein structures. Protein Data Bank Quarterly Newsletter 78, 10-11.

34. Murzin, A. G., Brenner, S. E., Hubbard, T. \& Chothia, C. (1995). SCOP: a structural classification of proteins database for the investigation of sequences and structures. $J$. Mol. Biol. 247, 536-540.

35. Kabsch, W., Kabsch, H. \& Eisenberg, D. (1976). Packing in a new crystalline form of glutamine synthetase from Escherichia coli. J. Mol. Biol. 100, 283-291.

36. Muller, P., Kopke, S. \& Sheldrick, G. M. (2003). Is the bond-valence method able to identify metal atoms in protein structures? Acta Crystallogr., Sect. D: Biol. Crystallogr. 59, 32-37.

37. Lee, B. \& Richards, F. M. (1971). The interpretation of protein structures: estimation of static accessibility. J. Mol. Biol. 55, 379-400.

38. (1994). The CCP4 suite: programs for protein crystallography. Acta Crystallogr., Sect. D: Biol.Crystallogr. 50, 760-763.

39. Potterton, E., McNicholas, S., Krissinel, E., Cowtan, K. \& Noble, M. (2002). The CCP4 molecular-graphics project. Acta Crystallogr., Sect. D: Biol.Crystallogr. 58, 1955-1957.

\section{FIGURE LEGENDS}

Fig. 1. Amino acid alignment of TvNiR, NrfAs, and multi-heme cytochromes performed with BLAST and ClustalW.

The second to fourth sequences have the highest level of identity to TvNiR (up to $53 \%$ ). The proteins with the known 3D structures are marked in blue. The background color code: gray, the signal peptide cleavage site (actual or predicted with SignalP 3.0); yellow, CXXCH or CXXCK motifs; red, conserved residues; orange, homologous residues; blue, residues involved in the substrate and product channels in NrfAs; cyan, the active site residues of NrfAs. The red and blue arrows indicate the residues involved in the substrate and product channels, respectively, of TvNiR, and cyan diamonds indicate the active site residues of TvNiR. For TvNiR, the sequence numbering starts with the first residue of the mature protein to coincide with that in PDB. The TrEMBL accession numbers of the sequences are: $\underline{\text { Q5F2I3 }}$ for Thioalkalivibrio nitratireducens; $\underline{\mathbf{Q 1 N Q Z 7}}$

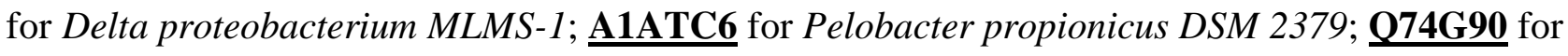
Geobacter sulfurreducens (8 hemes); Q747W1 for Geobacter sulfurreducens (5 hemes); $\underline{\mathbf{8 V N} \mathbf{2}}$ 
for Desulfovibrio desulfuricans; P0ABL0 for Escherichia coli; Q9Z4P4 for Sulfurospirillum

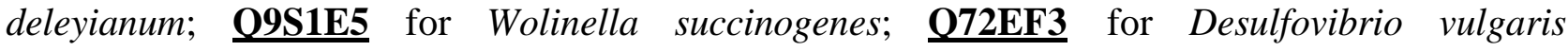
Hildenborough.

Fig. 2. TvNiR monomer

(a) Structure of the apo-TvNiR monomer. The N-terminal domain is shown in pink. In the catalytic domain the $\alpha$-helices are shown in yellow and the $\beta$-sheets in blue. The hemes, labeled sequentially from the $\mathrm{N}$ terminus, are green and the catalytic heme is red.

(b) Comparison of the monomers of TvNiR (left) and NrfA from W. succinogenes (right). The structurally equivalent elements located according to ${ }^{33}$ are shown in blue; the non-equivalent elements, in brown. The hemes in W. succinogenes are numbered in accordance with the heme-binding motifs in the corresponding sequence.

Fig. 3. TvNiR oligomeric structure

(a) Stereo view of the TvNiR hexamer. The monomers belonging to the same trimer are designated by the same letter, e.g., A1-A3. The monomers that build the respective dimers are shown in the same color and are denoted by the same numerical index, e.g., A1-B1. The twofold symmetry axis (horizontal) and the threefold symmetry axis (vertical) run in the plane of the figure.

(b) Stereo view of the inner cavity (gray surface) within the TvNiR trimer indicating the outlet of a product channel (blue surface). Only two monomers and one product channel are shown for clarity. The orientation is the same as in Fig. $3 a$.

(c) Charge distribution at the base of the TvNiR trimer. The threefold symmetry axis is perpendicular to the plane of the figure, and the twofold symmetry axis is in the plane of the figure. The hemes are shown in yellow. The negatively charged areas are shown in shades of red; the positively charged, in shades of blue. The putative donor binding sites are indicated by arrows.

(d) Charge distribution over the surface of the TvNiR hexamer. The orientation is the same as in Fig. 3a. The color scheme is the same as in Fig. 3c. The arrows point to hemes 8 belonging to different trimers.

Fig. 4. TvNiR active site

(a) Apo-TvNiR. Water molecules are shown as red spheres. 
(b) Complex with the substrate (nitrite); the Fo-Fc omit map is countoured at $3 \sigma$; the substrate and a water molecule occupy the same site at half occupancy.

(c) Complex with the inhibitor (azide); the Fo-Fc omit map is countoured at $3 \sigma$; the inhibitor and a water molecule occupy the same site at half occupancy.

(d) Apo-TvNiR. The electron density map (2Fo-Fc map, $1 \sigma$ ) showing the covalent bond between Cys305 and Tyr303.

Fig. 5. Charge distribution within the substrate/product channels in the TvNiR monomer

The catalytic heme is shown in red; other hemes, in green. The negatively charged areas are shown in shades of red; the positively charged areas, in shades of blue.

Fig. 6. Calcium-binding sites in the TvNiR monomer

(a) Primary $\mathrm{Ca}^{2+}$-binding site in the vicinity of the enzyme active site. The calcium ion is shown as a green sphere; water molecules, as red spheres.

(b) Secondary $\mathrm{Ca}^{2+}$-binding site in the vicinity of hemes 6 and 7 in the complex with nitrite. Only one conformation of the side chain of heme 7 involved in the binding of the $\mathrm{Ca}^{2+}$ ion is shown.

Fig. 7. Arrangement of hemes in TvNiR

(a) Superposition of the hemes in the TvNiR monomer (green) and the hemes of NrfA from W. succinogenes (red), HAO (blue), and OTR (yellow). The numbering scheme for the hemes corresponds to TvNiR.

(b) Arrangement of hemes in the TvNiR dimer. The catalytic hemes are shown in red. The twofold symmetry axis (horizontal) and the threefold symmetry axis (vertical) run in the plane of the figure.

(c) Arrangement of hemes in the TvNiR trimer. The threefold symmetry axis is perpendicular to the plane of the figure, and the twofold symmetry axis is in the plane of the figure.

Fig. 8. Model of binding of the NrfH dimer (yellow) to the TvNiR molecule (green). The A1 (left) and B2 (right) subunits are shown. The monomers in the $\mathrm{NrfH}$ dimer are related by the crystallographic twofold axis (vertical in the plane of the figure), which coincides with the non-crystallographic twofold axis that relates the A1 and B2 subunits of TvNiR. 